\title{
Infinitely many periodic solutions for subquadratic second-order Hamiltonian systems
}

Hua Gu* and Tianqing An

\section{"Correspondence:}

guhuasy@hhu.edu.cn

College of Science, Hohai University,

Nanjing, 210098, China

\section{Abstract}

In this paper, we investigate the existence of infinitely many periodic solutions for a class of subquadratic nonautonomous second-order Hamiltonian systems by using the variant fountain theorem.

\section{Introduction}

Consider the second-order Hamiltonian systems

$$
\left\{\begin{array}{l}
\ddot{u}(t)+\nabla_{u} W(t, u)=0, \quad \forall t \in \mathbb{R}, \\
u(0)=u(T), \quad \dot{u}(0)=\dot{u}(T), \quad T>0,
\end{array}\right.
$$

where $W(t, u)$ is also $T$-periodic and satisfies the following assumption (A):

(A) $W(t, u)$ is measurable in $t$ for all $u \in \mathbb{R}^{N}$, continuously differentiable in $u$ for a.e. $t \in[0, T]$ and there exist $a \in C\left(R^{+}, R^{+}\right)$and $b \in L^{1}\left([0, T], R^{+}\right)$such that

$$
|W(t, u)| \leq a(|u|) b(t), \quad\left|\nabla_{u} W(t, u)\right| \leq a(|u|) b(t)
$$

for all $u \in \mathbb{R}^{N}$ and a.e. $t \in[0, T]$.

Here and in the sequel, $\langle\cdot, \cdot\rangle$ and $|\cdot|$ always denote the standard inner product and the norm in $\mathbb{R}^{N}$ respectively.

There have been many investigations on the existence and multiplicity of periodic solutions for Hamiltonian systems via the variational methods (see [1-7] and the references therein). In [6], Zhang and Liu studied the asymptotically quadratic case of $W(t, u)=$ $\frac{1}{2}\langle U(t) u, u\rangle+W_{1}(t, u)$ under the following assumptions:

$\left(\mathrm{AQ}_{1}\right) W_{1}(t, u) \geq 0$ for all $(t, u) \in[0, T] \times \mathbb{R}^{N}$, and there exist constants $\mu \in(0,2)$ and $R_{1}>0$ such that

$$
\left\langle\nabla_{u} W_{1}(t, u), u\right\rangle \leq \mu W_{1}(t, u), \quad \forall t \in[0, T] \text { and }|u| \geq R_{1}
$$

$\left(\mathrm{AQ}_{2}\right) \lim _{|u| \rightarrow 0} \frac{W_{1}(t, u)}{|u|^{2}}=\infty$ uniformly for $t \in[0, T]$, and there exist constants $c_{2}, R_{2}>0$ such that

$$
W_{1}(t, u) \leq c_{2}|u|, \quad \forall t \in[0, T] \text { and }|u| \leq R_{2}
$$

( 2013 Gu and An; licensee Springer. This is an Open Access article distributed under the terms of the Creative Commons Attribution License (http://creativecommons.org/licenses/by/2.0), which permits unrestricted use, distribution, and reproduction in any medium, provided the original work is properly cited. 


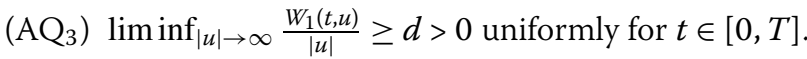

They obtained the existence of infinitely many periodic solutions of (1.1) provided $W_{1}(t, u)$ is even in $u$ (see Theorem 1.1 of [6]).

The subquadratic condition $\left(\mathrm{AQ}_{1}\right)$ is widely used in the investigation of nonlinear differential equations. This condition was weakened by some researchers; see, for example, [4] of Jiang and Tang. This paper considers the case of $U(t) \equiv 0$, then $W(t, u)=W_{1}(t, u)$. Motivated by $[4]$ and [6], we replace $\left(\mathrm{AQ}_{1}\right)$ with the following condition:

$\left(\mathrm{AQ}_{1}^{\prime}\right) W(t, u) \geq 0$ for all $(t, u) \in[0, T] \times \mathbb{R}^{N}$, and

$$
\begin{aligned}
& \lim _{|u| \rightarrow \infty}\left(\left\langle\nabla_{u} W(t, u), u\right\rangle-2 W(t, u)\right)=-\infty \text { and } \\
& \lim _{|u| \rightarrow \infty} \frac{W(t, u)}{|u|^{2}}=0 \quad \text { uniformly for } t \in[0, T] .
\end{aligned}
$$

The condition $\left(\mathrm{AQ}_{1}^{\prime}\right)$ implies that for some constant $R_{1}^{\prime}>0$,

$$
\left\langle\nabla_{u} W(t, u), u\right\rangle \leq 2 W(t, u), \quad \forall t \in[0, T] \text { and }|u| \geq R_{1}^{\prime} .
$$

By the assumption (A) and the condition $\left(\mathrm{AQ}_{1}^{\prime}\right)$, for any $\epsilon>0$, there exists a $\delta>0$ such that

$$
W(t, u) \leq \epsilon|u|^{2}+\max _{s \in[0, \delta]} a(s) b(t)
$$

for $\forall u \in \mathbb{R}^{N}$ and a.e. $t \in[0, T]$.

Meanwhile, we weaken the condition $\left(\mathrm{AQ}_{3}\right)$ to $\left(\mathrm{AQ}_{3}^{\prime}\right)$ as follows:

$\left(\mathrm{AQ}_{3}^{\prime}\right)$ There exists a constant $\varrho \in(0,1]$ such that

$$
\liminf _{|u| \rightarrow \infty} \frac{W(t, u)}{|u|^{e}} \geq d>0 \quad \text { uniformly for } t \in[0, T] .
$$

Then our main result is the following theorem.

Theorem 1.1 Assume that $\left(\mathrm{AQ}_{1}^{\prime}\right),\left(\mathrm{AQ}_{2}\right),\left(\mathrm{AQ}_{3}^{\prime}\right)$ hold and $W(t, u)$ is even in $u$. Then (1.1) possesses infinitely many solutions.

Remark The conditions $\left(\mathrm{AQ}_{1}\right)$ and $\left(\mathrm{AQ}_{3}\right)$ are stronger than $\left(\mathrm{AQ}_{1}^{\prime}\right)$ and $\left(\mathrm{AQ}_{3}^{\prime}\right)$. Then Theorem 1.1 above is different from Theorem 1.1 of [6].

\section{Preliminaries}

In this section, we establish the variational setting for our problem and give the variant fountain theorem. Let $E=H_{T}^{1}$ be the usual Sobolev space with the inner product

$$
\langle u, v\rangle_{E}=\int_{0}^{T}\langle u(t), v(t)\rangle d t+\int_{0}^{T}\langle\dot{u}(t), \dot{v}(t)\rangle d t .
$$

We define the functional on $E$ by

$$
\Phi(u)=\frac{1}{2} \int_{0}^{T}|\dot{u}|^{2} d t-\Psi(u)
$$


where $\Psi(u)=\int_{0}^{T} W(t, u(t)) d t$. Then $\Phi$ and $\Psi$ are continuously differentiable and

$$
\left\langle\Phi^{\prime}(u), v\right\rangle=\int_{0}^{T}\langle\dot{u}, \dot{v}\rangle d t-\int_{0}^{T}\left\langle\nabla_{u} W(t, u), v\right\rangle d t
$$

Define a self-adjoint linear operator $\mathcal{B}: L^{2}\left([0, T] ; \mathbb{R}^{N}\right) \rightarrow L^{2}\left([0, T] ; \mathbb{R}^{N}\right)$ by

$$
\int_{0}^{T}\langle\mathcal{B} u, v\rangle d t=\int_{0}^{T}\langle\dot{u}(t), \dot{v}(t)\rangle d t
$$

with the domain $D(\mathcal{B})=E$. Then $\mathcal{B}$ has a sequence of eigenvalues $\sigma_{k}=\frac{4 k^{2} \pi^{2}}{T^{2}}(k=0,1,2, \ldots)$. Let $\left\{e_{j}\right\}_{j=0}^{+\infty}$ be the system of eigenfunctions corresponding to $\left\{\sigma_{j}\right\}_{j=0}^{+\infty}$, it forms an orthogonal basis in $L^{2}$. Denote by $E^{+}=\left\{u \in E \mid \int_{0}^{T} u(t) d t=0\right\}, E^{0}=\mathbb{R}^{N}$, it is well known that

$$
\begin{aligned}
& E^{0}=\operatorname{ker} \mathcal{B}=\operatorname{span}\left\{e_{0}\right\}, \\
& E^{+}=\operatorname{span}\left\{e_{j} \mid j=1,2, \ldots\right\},
\end{aligned}
$$

and $E$ possesses orthogonal decomposition $E=E^{0} \oplus E^{+}$. For $u \in E$, we have

$$
u=u^{0}+u^{+} \in E^{0} \oplus E^{+}
$$

We can define on $E$ a new inner product and the associated norm by

$$
\langle u, v\rangle_{0}=\left\langle\mathcal{B} u^{+}, v^{+}\right\rangle_{L^{2}}+\left\langle u^{0}, v^{0}\right\rangle_{L^{2}},
$$

and

$$
\|u\|=\langle u, u\rangle_{0}^{\frac{1}{2}}
$$

Therefore, $\Phi$ can be written as

$$
\Phi(u)=\frac{1}{2}\left\|u^{+}\right\|^{2}-\Psi(u) .
$$

Direct computation shows that

$$
\begin{aligned}
& \left\langle\Psi^{\prime}(u), v\right\rangle=\int_{0}^{T}\left\langle\nabla_{u} W(t, u), v\right\rangle d t, \\
& \left\langle\Phi^{\prime}(u), v\right\rangle=\left\langle u^{+}, v^{+}\right\rangle_{0}-\left\langle\Psi^{\prime}(u), v\right\rangle
\end{aligned}
$$

for all $u, v \in E$ with $u=u^{0}+u^{+}$and $v=v^{0}+v^{+}$respectively. It is known that $\Psi^{\prime}: E \rightarrow E$ is compact.

Denote by $|\cdot|_{p}$ the usual norm of $L^{P}$, then there exists a $\tau_{p}>0$ such that

$$
|u|_{p} \leq \tau_{p}\|u\|, \quad \forall u \in E .
$$


We state an abstract critical point theorem founded in [8]. Let $E$ be a Banach space with the norm $\|\cdot\|$ and $E=\bigoplus_{j \in \mathbb{N}} X_{j}$ with $\operatorname{dim} X_{j}<\infty$ for any $j \in \mathbb{N}$. Set $Y_{k}=\bigoplus_{j=1}^{k} X_{j}$ and $Z_{k}=\overline{\bigoplus_{j=k}^{\infty} X_{j}}$. Consider the following $C^{1}$-functional $\Phi_{\lambda}: E \rightarrow \mathbb{R}$ defined by

$$
\Phi_{\lambda}(u):=A(u)-\lambda B(u), \quad \lambda \in[1,2] .
$$

Theorem 2.1 [8, Theorem 2.2] Assume that the functional $\Phi_{\lambda}$ defined above satisfies the following:

$\left(\mathrm{T}_{1}\right) \Phi_{\lambda}$ maps bounded sets to bounded sets uniformly for $\lambda \in[1,2]$, and $\Phi_{\lambda}(-u)=\Phi_{\lambda}(u)$ for all $(\lambda, u) \in[1,2] \times E$;

$\left(\mathrm{T}_{2}\right) \quad B(u) \geq 0$ for all $u \in E$, and $B(u) \rightarrow \infty$ as $\|u\| \rightarrow \infty$ on any finite-dimensional subspace of $E$;

$\left(\mathrm{T}_{3}\right)$ There exist $\rho_{k}>r_{k}>0$ such that

$$
\alpha_{k}(\lambda):=\inf _{u \in Z_{k},\|u\|=\rho_{k}} \Phi_{\lambda}(u) \geq 0>\beta_{k}(\lambda):=\max _{u \in Y_{k},\|u\|=r_{k}} \Phi_{\lambda}(u), \quad \forall \lambda \in[1,2]
$$

and

$$
\xi_{k}(\lambda):=\inf _{u \in Z_{k},\|u\| \leq \rho_{k}} \Phi_{\lambda}(u) \rightarrow 0 \quad \text { as } k \rightarrow \infty \text { uniformly for } \lambda \in[1,2] .
$$

Then there exist $\lambda_{n} \rightarrow 1, u_{\lambda_{n}} \in Y_{n}$ such that

$$
\left.\Phi_{\lambda_{n}}^{\prime}\right|_{Y_{n}}\left(u_{\lambda_{n}}\right)=0, \quad \Phi_{\lambda_{n}}\left(u_{\lambda_{n}}\right) \rightarrow \eta_{k} \in\left[\xi_{k}(2), \beta_{k}(1)\right] \text { as } n \rightarrow \infty
$$

Particularly, if $\left\{u_{\lambda_{n}}\right\}$ has a convergent subsequence for every $k$, then $\Phi_{1}$ has infinitely many nontrivial critical points $\left\{u_{k}\right\} \subset E \backslash\{0\}$ satisfying $\Phi_{1}\left(u_{k}\right) \rightarrow 0^{-}$as $k \rightarrow \infty$.

In order to apply this theorem to prove our main result, we define the functionals $A, B$ and $\Phi_{\lambda}$ on our working space $E$ by

$$
A(u)=\frac{1}{2}\left\|u^{+}\right\|^{2}, \quad B(u)=\int_{0}^{T} W(t, u) d t
$$

and

$$
\Phi_{\lambda}(u)=A(u)-\lambda B(u)=\frac{1}{2}\left\|u^{+}\right\|^{2}-\lambda \int_{0}^{T} W(t, u) d t
$$

for all $u=u^{0}+u^{+} \in E=E^{0}+E^{+}$and $\lambda \in[1,2]$. Then $\Phi_{\lambda} \in C^{1}(E, \mathbb{R})$ for all $\lambda \in[1,2]$. Let $X_{j}=\operatorname{span}\left\{e_{j}\right\}, j=0,1,2, \ldots$ Note that $\Phi_{1}=\Phi$, where $\Phi$ is the functional defined in (2.1).

\section{Proof of Theorem 1.1}

We firstly establish the following lemmas.

Lemma 3.1 Assume that $\left(\mathrm{AQ}_{1}^{\prime}\right)$ and $\left(\mathrm{AQ}_{3}^{\prime}\right)$ hold. Then $B(u) \geq 0$ for all $u \in E$ and $B(u) \rightarrow \infty$ as $\|u\| \rightarrow \infty$ on any finite-dimensional subspace of $E$. 
Proof Since $W(t, u) \geq 0$, by (2.4), it is obvious that $B(u) \geq 0$ for all $u \in E$.

By the proof of Lemma 2.6 of [6], for any finite-dimensional subspace $Y \subset E$, there exists a constant $\epsilon>0$ such that

$$
m(\{t \in[0, T]:|u| \geq \epsilon\|u\|\}) \geq \epsilon, \quad \forall u \in Y \backslash\{0\}
$$

where $m(\cdot)$ is the Lebesgue measure.

For the $\epsilon$ given in (3.1), let

$$
\Lambda_{u}=\{t \in[0, T]:|u| \geq \epsilon\|u\|\}, \quad \forall u \in Y \backslash\{0\}
$$

Then $m\left(\Lambda_{u}\right) \geq \epsilon$. By $\left(\mathrm{AQ}_{3}^{\prime}\right)$, there exists a constant $R_{3}>R_{1}^{\prime}$ such that

$$
W(t, u) \geq d|u|^{\varrho} / 2, \quad \forall t \in[0, T] \text { and }|u| \geq R_{3},
$$

where $R_{1}^{\prime}$ is the constant given in (1.2). Note that

$$
|u(t)| \geq R_{3}, \quad \forall t \in \Lambda_{u}
$$

for any $u \in Y$ with $\|u\| \geq R_{3} / \epsilon$. Thus,

$$
\begin{aligned}
B(u) & =\int_{0}^{T} W(t, u) d t \geq \int_{\Lambda_{u}} W(t, u) d t \geq \int_{\Lambda_{u}} d|u|^{\varrho} / 2 d t \\
& \geq d \epsilon^{\varrho}\|u\|^{\varrho} \cdot m\left(\Lambda_{u}\right) / 2 \geq d \epsilon^{\varrho+1}\|u\|^{\varrho} / 2
\end{aligned}
$$

for any $u \in Y$ with $\|u\| \geq R_{3} / \epsilon$. This implies $B(u) \rightarrow \infty$ as $\|u\| \rightarrow \infty$ on $Y$.

Lemma 3.2 Assume that $\left(\mathrm{AQ}_{1}^{\prime}\right),\left(\mathrm{AQ}_{2}\right)$ and $\left(\mathrm{AQ}_{3}^{\prime}\right)$ hold. Then there exist a positive integer $k_{1}$ and two sequences $0<r_{k}<\rho_{k} \rightarrow 0$ as $k \rightarrow \infty$ such that

$$
\begin{aligned}
& \alpha_{k}(\lambda):=\inf _{u \in Z_{k},\|u\|=\rho_{k}} \Phi_{\lambda}(u)>0, \quad \forall k \geq k_{1}, \\
& \xi_{k}(\lambda):=\inf _{u \in Z_{k},\|u\| \leq \rho_{k}} \Phi_{\lambda}(u) \rightarrow 0 \quad \text { as } k \rightarrow \infty \text { uniformly for } \lambda \in[1,2],
\end{aligned}
$$

and

$$
\beta_{k}(\lambda):=\max _{u \in Y_{k},\|u\|=r_{k}} \Phi_{\lambda}(u)<0, \quad \forall k \in \mathbb{N}
$$

where $Y_{k}=\bigoplus_{j=0}^{k} X_{j}=\operatorname{span}\left\{e_{0}, e_{1}, \ldots, e_{k}\right\}$ and $Z_{k}=\overline{\bigoplus_{j=k}^{\infty} X_{j}}=\overline{\operatorname{span}\left\{e_{k}, e_{k+1}, \ldots\right\}}$ for all $k \in \mathbb{N}$.

Proof Comparing this lemma with Lemma 2.7 of [6], we find that these two lemmas have the same condition $\left(\mathrm{AQ}_{2}\right)$ which is the key in the proof of Lemma 2.7 of [6]. We can prove our lemma by using the same method of [6], so the details are omitted.

Now it is the time to prove our main result Theorem 1.1. 
Proof of Theorem 1.1 By virtue of (1.3), (2.3) and (2.5), $\Phi_{\lambda}$ maps bounded sets to bounded sets uniformly for $\lambda \in[1,2]$. Obviously, $\Phi_{\lambda}(-u)=\Phi_{\lambda}(u)$ for all $(\lambda, u) \in[1,2] \times E$ since $W(t, u)$ is even in $u$. Consequently, the condition $\left(\mathrm{T}_{1}\right)$ of Theorem 2.1 holds. Lemma 3.1 shows that the condition $\left(\mathrm{T}_{2}\right)$ holds, whereas Lemma 3.2 implies that the condition $\left(\mathrm{T}_{3}\right)$ holds for all $k \geq k_{1}$, where $k_{1}$ is given there. Therefore, by Theorem 2.1 , for each $k \geq k_{1}$, there exist $\lambda_{n} \rightarrow 1$ and $u_{\lambda_{n}} \in Y_{n}$ such that

$$
\left.\Phi_{\lambda_{n}}^{\prime}\right|_{Y_{n}}\left(u_{\lambda_{n}}\right)=0, \quad \Phi_{\lambda_{n}}\left(u_{\lambda_{n}}\right) \rightarrow \eta_{k} \in\left[\xi_{k}(2), \beta_{k}(1)\right] \quad \text { as } n \rightarrow \infty
$$

For the sake of notational simplicity, in the following we always set $u_{n}=u_{\lambda_{n}}$ for all $n \in \mathbb{N}$.

Step 1 . We firstly prove that $\left\{u_{n}\right\}$ is bounded in $E$.

Since $\left\{u_{n}\right\}$ satisfies (3.7), one has

$$
\lim _{n \rightarrow \infty}\left(\left\langle\left.\Phi_{\lambda_{n}}^{\prime}\right|_{Y_{n}}\left(u_{n}\right), u_{n}\right\rangle-2 \Phi_{\lambda_{n}}\left(u_{n}\right)\right)=-2 \eta_{k}
$$

More precisely,

$$
\lim _{n \rightarrow \infty} \int_{0}^{T}\left(\left\langle\nabla_{u} W\left(t, u_{n}\right), u_{n}\right\rangle-2 W\left(t, u_{n}\right)\right) d t=2 \eta_{k}
$$

Now, we prove that $\left\{u_{n}\right\}$ is bounded. Otherwise, without loss of generality, we may assume that

$$
\left\|u_{n}\right\| \rightarrow \infty \quad \text { as } n \rightarrow \infty
$$

Put $z_{n}=\frac{u_{n}}{\left\|u_{n}\right\|}$, we have $\left\|z_{n}\right\|=1$. Going to a subsequence if necessary, we may assume that

$$
z_{n} \rightarrow z \quad \text { in } E, \quad z_{n} \rightarrow z \quad \text { in } L^{2} \quad \text { and } \quad z_{n}(t) \rightarrow z(t) \quad \text { for a.e. } t \in[0, T] \text {. }
$$

By (1.3), we have

$$
\begin{aligned}
\Phi_{\lambda_{n}}\left(u_{n}\right) & =\frac{1}{2}\left\|u_{n}^{+}\right\|^{2}-\lambda_{n} \int_{0}^{T} W\left(t, u_{n}\right) d t \\
& \geq \frac{1}{2}\left\|u_{n}\right\|^{2}-\frac{1}{2}\left\|u_{n}^{0}\right\|^{2}-\lambda_{n}\left(\epsilon \int_{0}^{T}\left|u_{n}\right|^{2} d t+\max _{s \in[0, \delta]} a(s) \int_{0}^{T} b(t) d t\right) \\
& \geq \frac{1}{2}\left\|u_{n}\right\|^{2}-\left(\frac{1}{2}+\lambda_{n} \epsilon\right) \int_{0}^{T}\left|u_{n}\right|^{2} d t-\lambda_{n} c_{1},
\end{aligned}
$$

where $c_{1}=\max _{s \in[0, \delta]} a(s) \int_{0}^{T} b(t) d t$. Therefore, one obtains

$$
\begin{aligned}
\frac{\Phi_{\lambda_{n}}\left(u_{n}\right)}{\left\|u_{n}\right\|^{2}} & \geq \frac{1}{2}-\left(\frac{1}{2}+\lambda_{n} \epsilon\right) \int_{0}^{T}\left(\frac{\left|u_{n}\right|}{\left\|u_{n}\right\|}\right)^{2} d t-\frac{\lambda_{n} c_{1}}{\left\|u_{n}\right\|^{2}} \\
& =\frac{1}{2}-\left(\frac{1}{2}+\lambda_{n} \epsilon\right)\left\|z_{n}\right\|_{2}^{2}-\frac{\lambda_{n} c_{1}}{\left\|u_{n}\right\|^{2}} .
\end{aligned}
$$


Passing to the limit in the inequality, by using $\Phi_{\lambda_{n}}\left(u_{n}\right) \rightarrow \eta_{k}$ and $\lambda_{n} \rightarrow 1$ as $n \rightarrow \infty$, we obtain

$$
\frac{1}{2}-\left(\frac{1}{2}+\epsilon\right)\|z\|_{2}^{2} \leq 0
$$

Thus, $z \neq 0$ on a subset $\Omega$ of $[0, T]$ with positive measure.

By (1.2), we have

$$
\left\langle\nabla_{u} W(t, u), u\right\rangle-2 W(t, u) \leq 0, \quad \forall t \in[0, T] \text { and }|u| \geq R_{1}^{\prime},
$$

and by the assumption (A), we obtain

$$
\left\langle\nabla_{u} W(t, u), u\right\rangle-2 W(t, u) \leq c_{3} b(t), \quad \text { for all }|u| \leq R_{1}^{\prime} \text { and a.e. } t \in[0, T]
$$

where $c_{3}=\left(2+R_{1}^{\prime}\right) \max _{\left[0, R_{1}^{\prime}\right]} a(s)$. So, we get

$$
\left\langle\nabla_{u} W(t, u), u\right\rangle-2 W(t, u) \leq c_{3} b(t)
$$

for all $u \in \mathbb{R}^{N}$ and a.e. $t \in[0, T]$. Hence,

$$
\begin{aligned}
& \int_{0}^{T}\left(\left\langle\nabla_{u} W\left(t, u_{n}\right), u_{n}\right\rangle-2 W\left(t, u_{n}\right)\right) d t \\
& \quad=\int_{\Omega}\left(\left\langle\nabla_{u} W\left(t, u_{n}\right), u_{n}\right\rangle-2 W\left(t, u_{n}\right)\right) d t+\int_{[0, T] \backslash \Omega}\left(\left\langle\nabla_{u} W\left(t, u_{n}\right), u_{n}\right\rangle-2 W\left(t, u_{n}\right)\right) d t \\
& \quad \leq \int_{\Omega}\left(\left\langle\nabla_{u} W\left(t, u_{n}\right), u_{n}\right\rangle-2 W\left(t, u_{n}\right)\right) d t+\int_{[0, T] \backslash \Omega} c_{3} b(t) d t .
\end{aligned}
$$

An application of Fatou's lemma yields

$$
\int_{\Omega}\left(\left\langle\nabla_{u} W\left(t, u_{n}\right), u_{n}\right\rangle-2 W\left(t, u_{n}\right)\right) d t \rightarrow-\infty \quad \text { as } n \rightarrow \infty,
$$

which is a contradiction to (3.8).

Step 2. We prove that $\left\{u_{n}\right\}$ has a convergent subsequence in $E$.

Since $\left\{u_{n}\right\}$ is bounded in $E, E$ is reflexible and $\operatorname{dim} E^{0}<\infty$, without loss of generality, we assume

$$
u_{n}^{0} \rightarrow u_{0}^{0}, \quad u_{n}^{+} \rightarrow u_{0}^{+} \quad \text { and } \quad u_{n} \rightarrow u_{0} \quad \text { as } n \rightarrow \infty
$$

for some $u_{0}=u_{0}^{0}+u_{0}^{+} \in E=E^{0} \oplus E^{+}$.

Note that

$$
0=\left.\Phi_{\lambda_{n}}^{\prime}\right|_{Y_{n}}\left(u_{n}\right)=u_{n}^{+}-\lambda_{n} P_{n} \Psi^{\prime}\left(u_{n}\right), \quad \forall n \in \mathbb{N},
$$

where $P_{n}: E \rightarrow Y_{n}$ is the orthogonal projection for all $n \in \mathbb{N}$, that is,

$$
u_{n}^{+}=\lambda_{n} P_{n} \Psi^{\prime}\left(u_{n}\right), \quad \forall n \in \mathbb{N} .
$$


In view of the compactness of $\Psi^{\prime}$ and (3.9), the right-hand side of (3.10) converges strongly in $E$ and hence $u_{n}^{+} \rightarrow u_{0}^{+}$in $E$. Together with (3.9), we have $u_{n} \rightarrow u_{0}$ in $E$.

Now, from the last assertion of Theorem 2.1, we know that $\Phi=\Phi_{1}$ has infinitely many nontrivial critical points. The proof is completed.

Competing interests

The authors declare that they have no competing interests.

Authors' contributions

HG wrote the first draft and TA corrected and improved the final version. All authors read and approved the final draft.

\section{Acknowledgements}

The authors thank the referee for his/her careful reading of the manuscript. The work is supported by the Fundamental Research Funds for the Central Universities and the National Natural Science Foundation of China (No. 61001139).

Received: 8 November 2012 Accepted: 10 January 2013 Published: 6 February 2013

\section{References}

1. Chen, G, Ma, S: Periodic solutions for Hamiltonian systems without Ambrosetti-Rabinowitz condition and spectrum 0 . J. Math. Anal. Appl. 379, 842-851 (2011)

2. Ding, Y, Lee, C: Periodic solutions for Hamiltonian systems. SIAM J. Math. Anal. 32, 555-571 (2000)

3. He, X, Wu, X: Periodic solutions for a class of nonautonomous second order Hamiltonian systems. J. Math. Anal. Appl. $341(2), 1354-1364(2008)$

4. Jiang, Q, Tang, C: Periodic and subharmonic solutions of a class of subquadratic second-order Hamiltonian systems. J. Math. Anal. Appl. 328, 380-389 (2007)

5. Wang, Z, Zhang, J: Periodic solutions of a class of second order non-autonomous Hamiltonian systems. Nonlinear Anal. 72, 4480-4487 (2010)

6. Zhang, Q, Liu, C: Infinitely many periodic solutions for second-order Hamiltonian systems. J. Differ. Equ. 251, 816-833 (2011)

7. Zou, W: Multiple solutions for second-order Hamiltonian systems via computation of the critical groups. Nonlinear Anal. TMA 44, 975-989 (2001)

8. Zou, W: Variant fountain theorems and their applications. Manuscr. Math. 104, 343-358 (2001)

doi:10.1186/1687-2770-2013-16

Cite this article as: Gu and An: Infinitely many periodic solutions for subquadratic second-order Hamiltonian systems. Boundary Value Problems 2013 2013:16

\section{Submit your manuscript to a SpringerOpen ${ }^{\circ}$ journal and benefit from:}

- Convenient online submission

- Rigorous peer review

- Immediate publication on acceptance

- Open access: articles freely available online

- High visibility within the field

- Retaining the copyright to your article 